\title{
Remarks about B Production in pA Collisions with a p-Beam of $8 \mathrm{TeV}$
}

\author{
A. Fridman* and A. Penzo ${ }^{+}$ \\ CERN, Geneva, Switzerland
}

\begin{abstract}
We discuss $p A$ collisions that can be used for studying $B$-physics with a proton beam of $\sim 8 \mathrm{TeV} / \mathrm{c}$. In particular we study $R(A)=\sigma^{A}(b \bar{b}) / \sigma_{T}^{A}$ as well as the number of interactions per bunch crossing of the beam with the various targets. We also estimate the average charged multiplicity for $p A$ collisions. Finally some comments are made for the case of using external or gas jet targets.
\end{abstract}

Submitted to the 3th Topical Seminar on

Heavy Flavor, San Miniato, 17-21 June 1991

* LPNHE, Université de Paris VI et VII

+ INFN - Trieste 


\section{1 - Introduction}

In this note we discuss the kind of target that can be used in order to study $B$-physics with a proton beam having a momentum of $8 \mathrm{Tev} / \mathrm{c}$. We will discuss the value of the ratio

$$
R(A)=\frac{\sigma^{A}(b \bar{b})}{\sigma_{T}^{A}}
$$

The $A$ value is the target nuclear mass number (often called the atomic number corresponding to the number of nucleons in the target) whereas the $T$ index indicates the total inelastic case. Thus, $\sigma_{T}^{A}$ and $\sigma^{A}(b \bar{b})$ are the inelastic and $b \bar{b} X$ cross sections, respectively ( $X$ meaning anything). In principle, the possibility of increasing $R(A)$ may decrease the background among the event.s used for studying $B-\bar{B}$ physics. This might be very helpful for $p N$ interactions with an $8 \mathrm{TeV}$ beam where the background is estimated to be important (see below). We will also estimate the number of interactions per bunch crossing of the bearn with the various targets as well as the average charged multiplicity for $p A$ collisions.

For the time being the experimental data do not allow an estimate of $R(A)$ for $B \bar{B}$ production. Therefore, the study of charm and $\Upsilon(n S)$ production may suggest values for the $B \bar{B}$ case. In the next section we will discuss some features related to the $R(A)$ quantity. Then we will examine the charm and $\Upsilon(n S)$ production (Section 3). Finally we will make some remarks about the nuclear targets that can be used for studying $B$-physics (Section 4).

\section{2 - The $\mathbf{R}(\mathbf{A})$ dependance}

Usually the dependance of the inelastic and hadroproduction cross sections on a nuclear target with a given incident beam particle type are parametrized by $\sigma \sim A^{\alpha}$. Here $\alpha$ is a parameter which can be determined experimentally. In fact one assumes that with the same type of beam one has:

$$
\begin{aligned}
\sigma^{A}(h) & =\sigma^{0}(h) A^{\alpha(h)} \\
\sigma_{T}^{A} & =\sigma_{T}^{0} A^{\alpha(T)}
\end{aligned}
$$

where $h$ is the particle observed in the inclusive reaction. Here $\sigma_{T}^{0}, \sigma^{0}(h)$ denote parameters close to the $\sigma_{T}$ and $\sigma(h)$ cross sections of the beam on a proton target (the beam- $p$ and beam- $n$ cross section are assumed to be equal). The experimental data show that the above hadronic cross sections are well determined by the $A^{\alpha}$ parametrization for $A \geq 4$. With an hydrogen target the data tend to be somewhat 
below such a fit ${ }^{1}$. One also compares the results obtained with two different targets $\left(A_{1}, A_{2}\right)$ by using

$$
E \frac{d^{3} \sigma^{A_{2}}(h)}{d p^{3}}=E \frac{d^{3} \sigma^{A_{1}}(h)}{d p^{3}} \times\left(\frac{A_{2}}{A_{1}}\right)^{\alpha(h)}
$$

whereas $E, p$ are the energy and momentum of the outgoing $h$ particle. Nevertheles, in order to estimate

$$
R(A)=\frac{\sigma^{A}(h)}{\sigma_{T}^{A}}=\frac{\sigma^{0}(h)}{\sigma_{T}^{0}} A^{\alpha(h)-\alpha(T)}
$$

we will assume that $\sigma^{0}(h) / \sigma_{T}^{0}=\sigma(h) / \sigma_{T}$.

If one assumes that the total cross section is proportional to the surface of the nucleus target, $\alpha(T) \sim 2 / 3$ (see for instance Ref. 2). However, the $A$ dependence of $\sigma_{T}$ for $\pi^{ \pm}, K^{ \pm}, p$ and $\bar{p}$ beams have been investigated ${ }^{3}$ for incident momentum of $60-280 \mathrm{GeV} / \mathrm{c}$. For $p$ beams it was found that

$$
\alpha(T)=0.72 \pm 0.01
$$

while another $p A$ experiment ${ }^{4}$ at $400 \mathrm{GeV} / \mathrm{c}$ gives

$$
\alpha(T)=0.77 \pm 0.03
$$

For the present discussion we will use $\alpha(T)=0.72$, a value which is usually taken for beams with large incident momentum ${ }^{5}$.

According to the QCD model, $\alpha(h)$ is expected to be $\sim 1$ (Ref. 6) for $h$ particles containing heavy quarks (charm or bottom). Coherent effects may decrease the transparency of the nucleus and $\alpha(h)$ could then be reduced. Experimentally, one has found that $\alpha(h)$ depends on $x_{F}$ and $p_{T}$. Here $x_{F}$ is the Feynman scaling variable (in the beam-nucleon $\mathrm{cm}$ system) of the $h$ particle while $p_{T}$ is its transverse momentum (the Fermi motion of the nucleon inside the target is usually neglected). Thus, one has observed that for hadroproduction ${ }^{7,1}$ :

- $\alpha$ increases with $p_{T}$

$-\alpha$ increases when $x_{F}$ decreases 
Fig. 1 presents some results of various experiments ${ }^{9-11}$. In the region where $x_{F}$ and $p_{T}$ have large values the number of events is rather small as the inclusive differential cross section can be parametrized by ${ }^{8,12}$

$$
\frac{d^{2} \sigma}{d x_{F} d p_{T}^{2}} \sim\left(1-\left|x_{F^{\prime}}\right|\right)^{n} f\left(p_{T}\right)
$$

Here $f\left(p_{T}\right)$ is taken as

$$
e^{-b_{2} p_{T}^{2}} \quad \text { or } \quad e^{-b_{1} p_{T}}
$$

(Ref. 8), $n$ and $b_{1,2}$ being fitted parameters. In principle $n$ is related to the parton structure functions. For instance, $\pi^{-} A$ interactions with a beam of $340 \mathrm{GeV} / \mathrm{c}$ leads for charm production to ${ }^{8}, n=3.40 \pm 0.45$ and $b_{1}=2.00 \pm 0.12 \mathrm{GeV} / \mathrm{c}^{-1}$ whereas for $p p$ interactions ${ }^{13}$ it was found that $3 \leq n \leq 12$. In any case one sees from equation (1) that the average $\alpha$ value will not vary very much if the experiment will not be able to detect events with large $p_{T}$ or $x_{F}$.

From some experimental results ${ }^{3}$ one observed that for a given type of beam, $\alpha(h)$ tends to increase with the mass of the $h$ particle. This behaviour is not confirmed by reference (1). However, a recent experiment with a $340 \mathrm{GeV} / \mathrm{c} \pi^{-}$ beam $^{8}$ (WA82 charm production) has measured

$$
\begin{aligned}
\alpha\left(K_{S}^{0}\right) & =0.70 \pm 0.005 \pm 0.05,\left\langle x_{F}>=0.05\right. \\
\alpha(D) & =0.89 \pm 0.05 \pm 0.05, \quad\left\langle x_{F}\right\rangle=0.2
\end{aligned}
$$

According to our discussion $\alpha(D)$ will tend to be larger if $\left\langle x_{F}\right\rangle$ will have the lower value used for the $\alpha\left(K_{S}^{0}\right)$ case. Thus, one sees again that $\alpha(h)$ tend to increase with the mass of the observed outgoing particle.

\section{3 - Charm and beauty production}

For the time being the experimental data do not allow to estimate $R(A)$ for $B / \bar{B}$ production with $A$ targets. As mentioned above the study of the $D, \bar{D}$, and $J / \psi$ or $\psi^{\prime}$ production may indicate a lower limit of $\alpha(b)$ for the outgoing $B / \bar{B}$. Table 1 and 2 give the $\alpha$ values found for the $D / \bar{D}$ and $\psi$ production. A recent experiment ${ }^{22}$ has also studied the $\Upsilon, \Upsilon^{\prime}$ and $\Upsilon^{\prime \prime}$ production in $p A$ collisions at a $p$ momentum of $800 \mathrm{GeV} / \mathrm{c}$ (Table 2). As expected the $\alpha(\Upsilon)$ appear to be rather large, namely $\sim 0.95$. 
For the charm production there might be differences between samples heaving leading and non leading ${ }^{23} D / \bar{D}$. For instance, the $\pi^{-} p$ reactions at $340 \mathrm{GeV} / \mathrm{c}$ indicates ${ }^{24}$ that the $x_{F}$ distribution for the non leading charm mesons has more events for small $x_{F}$ than the sample containing leading charm mesons. This effect has not been confirmed by recent results ${ }^{8}$. For the present discussion we will not consider the possibility that $\alpha$ might depend from the presence of leading meson in the final state. In any case this problem does not exist for the $J / \psi, \psi^{\prime}$ or $\Upsilon(n S)$ production.

From Table 1 one sees that $\alpha(D)$ appears to be in the $0.76-0.97$ range, the errors of the experimental results being rather large. For the $J / \psi$ production (Table 2) the values tend to be larger than most of those given in Table 1 . The large $\alpha(J / \psi)$ values determined with the $\mathrm{H}$ and $\mathrm{Pt}$ targets $^{19}$ might also be due to the fact that the cross sections on proton are below the fits obtained with $A \geq 4$. However, the recent results ${ }^{21,22}$ obtained with large statistics indicate that $\alpha(J / \psi) \simeq 0.92$ and $\alpha(\Upsilon) \sim 0.95$. In any case new experiments will certainly help to clarify the values of the $\alpha$ parameter for charm and beauty production.

Let us now comment the $p N \rightarrow B \bar{B} X$ reactions resulting from a proton beam interacting with a fixed target. For the LHC project ( $p$ beam of $8 \mathrm{TeV}$ ) there are theoretical predictions ${ }^{25,26}$ for the total $\left(\sigma_{T}\right)$ and $b \bar{b} X(\sigma(b \bar{b}))$ cross sections, namely $\sigma_{T} \sim 50 \mathrm{mb}$ and $\sigma(b \bar{b}) \sim 1 \mu \mathrm{b}$ yielding

$$
R(A=1)=\frac{\sigma(b \tilde{b})}{\sigma_{T}} \sim \frac{1}{50000}
$$

In other words such a ratio indicate that 50000 inelastic interactions have to occur in order to have $1 B \bar{B}$ event. The tagging, the detection efficiencies as well as the triggering method for a specific experiment will, of course, decrease the non- $B \vec{B}$ events (the background events). This could only be estimated by defining:

- the type of $B$ decays which will be studied

- the detector properties.

One has, however, to emphasize that for the $p p$ collider one xnay have larger values, namely

$$
R(A=1) \sim \frac{1}{550} \sim \frac{1}{200}
$$

for the LHC or SSC projects ${ }^{27}$, respectively. For the $p A$ collisions we estimate 
$R(A)=\sigma(b \bar{b}) / \sigma_{T} F(A)$ with

$$
F(A)=A^{\alpha(B)-0.72}
$$

and using the predicted $p N$ cross sections. With the range of $0.9 \leq \alpha(B) \leq 1$, corresponding to the $\alpha(D), \alpha(J / \psi)$ and $\alpha(\Upsilon)$ values, one obtains estimates of $F(A)$ (See the scheme shown in Fig. 2). Clearly the utilization of $A$ targets could only be useful if $\alpha(B) \geq 0.9$ and $A \geq 80$. In these cases $R(A)$ will increase by a factor of $\sim 2.5$ with respect to the $A=1$ case.

\section{4 - The A targets for $B$ production}

For an LHC type of project, there are difficulties to build an external $p$ beam of $\sim 8 \mathrm{TeV} / \mathrm{c}$. In the case that such an eventual beam could have an intensity of $\sim 10^{8} \mathrm{p} / \mathrm{s}$, one obtains the luminosities given in Table 3 for various $A$ targets. For the present example we used targets with a length of $0.2 \mathrm{~cm}$ (along the beam direction). Table 3 gives also the number of $b \bar{b} X$ events in 1 year $\left(10^{7} s\right)$ of running $(N(b \bar{b}) / y)$, the number of inelastic interactions per second $\left(N_{\text {int }} / s\right)$ as well as the $R(A)$ ratio calculated with $\alpha_{T}=0.72$ and $\alpha(B)=1$. We note that the $N(b \bar{b}) / y$ are much smaller than those expected for the LHC $\left(\sim 210^{11} / y\right)$ or the SSC $\left(\sim 510^{11} / y\right)$ collider projects ${ }^{27}$. Assuming hete that an external beam would have the same structure form as in the collider, one may estimate the multiple interactions per bunch crossing with the fixed target. This might be done by estimating the $P(2) / P(1)$ and $P(3) / P(1)$ ratios. Here $P(m)$ is the probability of having $m$ interactions per bunch crossing and assumed to follow a Poisson distribution. We see from Table 3 that these effects are small $\left(\leq 2.510^{-2}\right)$. Note that in order to increase $N(b \bar{b}) / y$, one can also consider several target planes ${ }^{5,8}$ (each plane has then the same values of $P(m) / P(1)$ and $R(A)$ ). In this case one has to take into account the secondary interactions introduced by the outgoing particles.

In principle the gas jet target has the possibility of having large luminosities as the beam intensity in the $\mathrm{LHC}$ collider project is expected to be $\sim 5.410^{18} \mathrm{p} / \mathrm{s}$. The luminosity has, however, to be limited if one wants to avoid multiple interactions per bunch crossing. In Table 4 we calculate the luminosities for the various $A$ targets in order to have $P(2) / P(1)=0.05$ using a gas jet target with a length of $0.8 \mathrm{~cm}$ along the beam direction ${ }^{28}$. This correspond to $N_{\text {int }} / \mathrm{s}=5.510^{6}$. It would certainly be useful to also investigate the possibility of using several gas jet targets in a given experiment ${ }^{29}$. For instance, with three Xe gas jet target, one could eventually obtain $\sim 10^{10} b \bar{b} X$ events per year. Clcarly a detailed study has to be done in order to estimate the real number of $B \bar{B}$ that could be detected. 
The average charged multiplicity on a nuclear target $(<n(p A)\rangle)$ is expected to be larger than that obtained in $p p$ collisions $(<n(p p)>)$. The experimental data indicate ${ }^{30}$ that

$$
r=\frac{\langle n(p A)\rangle}{\langle n(p p)\rangle} \simeq \frac{1+\ddot{\nu}}{2} .
$$

Here $\bar{\nu}$ is the average number of collisions of the incident particle in the nuclear target which can be estimated by ${ }^{30}$

$$
\bar{\nu}=\frac{A \sigma_{T}}{\sigma_{T}^{A}}
$$

The $r$ values calculated by this method are given in Table 3 and 4 . We see that the $<(n(p A)\rangle$ values are not much larger than $\langle n(p p)\rangle$ (about 17 at the considered cm energy $\left.{ }^{27}\right)$.

To summarize, we discussed the $R(A)=\sigma^{A}(b \bar{b}) / \sigma_{T}^{A}$ quantity estimated by using experimental results of $D, J / \psi$ and $\Upsilon(n S)$ produced in hadron- $A$ collisions. Within the QCD approach, it appears that for $A \geq 80, R(A)$ will increase by a factor of $\sim 3$ with respect to $R(A=1)$ corresponding to $p p$ collisions. Thus the utilization of $A \geq 80$ targets might be useful to increase the signal to background ratio in the $B \bar{B}$ samples. We also estimated the $\langle n(p A)\rangle$ values which appear to be larger by a factor of 2 to 3 with respect to $p p$ interactions. Further detailed analyses are necessary in order to appreciate the real usefulness of $A$-targets with realistic detectors.

\section{Acknowledgments}

It is a pleasure to thank P. Hoycr, Y. Lemoigne, L. R.ossi and M.J. Tannenbaum for useful discussions. 
Table 1 - Some $\alpha$ values obtained from charm production. We took only experiments where the beam momentun is larger $100 \mathrm{GeV} / \mathrm{c}$.

\begin{tabular}{|c|c|c|c|c|}
\hline $\begin{array}{l}\text { beam } \\
\mathrm{GeV} / \mathrm{c}\end{array}$ & target & $\begin{array}{c}\text { some } \\
\text { details }\end{array}$ & $\alpha$ & reference \\
\hline$\pi^{-}, 320$ & $\mathrm{Al}, \mathrm{Fe}, \mathrm{U}$ & prompt $\mu^{ \pm}$ & $\begin{array}{l}\alpha\left(\mu^{+}\right)=0.76 \pm 0.08 \\
\alpha\left(\mu^{-}\right)=0.83 \pm 0.06\end{array}$ & 14 \\
\hline$p, 300$ & $\mathrm{Al}, \mathrm{Fe}, \mathrm{U}$ & $\begin{array}{c}\text { prompt } \mu^{ \pm} \\
x_{F}>0.1\end{array}$ & $\begin{array}{l}\alpha\left(\mu^{+}\right)=0.79 \pm 0.12 \\
\alpha\left(\mu^{-}\right)=0.76 \pm 0.13\end{array}$ & 15 \\
\hline$p, 400$ & $\mathrm{Be}, \mathrm{Cu}, \mathrm{W}$ & prompt $\left(\nu_{e}+\bar{\nu}_{e}\right)$ & $0.75 \pm 0.05$ & 16 \\
\hline$\pi^{ \pm}, 250$ & $\begin{array}{c}\mathrm{Be}, \mathrm{Al}, \mathrm{Cu}, \\
\mathrm{We}\end{array}$ & & $\begin{array}{l}\alpha\left(D^{+}\right)=0.97 \pm 0.07 \\
\alpha\left(D^{0}\right)=0.92 \pm 0.08\end{array}$ & 17 \\
\hline$\pi^{ \pm}, 250$ & $\mathrm{Si}, \mathrm{W}$ & $\left\langle x_{F}\right\rangle=0.24$ & $\alpha(D)=0.88 \pm 0.05$ & 18 \\
\hline
\end{tabular}

Table 2 - Same as in Table 1 but for $J / \psi, \psi^{\prime}$ and $\Upsilon(n S)$ production.

\begin{tabular}{|c|c|c|c|c|}
\hline $\begin{array}{l}\text { beam } \\
\mathrm{GeV} / \mathrm{c}\end{array}$ & target & $\begin{array}{l}\text { some } \\
\text { details }\end{array}$ & $\alpha$ & reference \\
\hline $\begin{array}{l}\pi^{-}, 150 \\
\pi^{-}, 200 \\
\pi^{-}, 280 \\
\pi^{+}, 200 \\
p, 200\end{array}$ & $\mathrm{H}, \mathrm{Pt}$ & $\begin{array}{c}J / \psi \\
\mu^{+} \mu^{-} \\
x_{F}>0\end{array}$ & $\begin{array}{l}0.95 \pm 0.02 \\
0.97 \pm 0.02 \\
0.95 \pm 0.02 \\
0.96 \pm 0.02 \\
0.94 \pm 0.03\end{array}$ & 19 \\
\hline$\pi^{-}, 530$ & $\begin{array}{c}\mathrm{C}, \mathrm{Al}, \mathrm{Cu} \\
\mathrm{Pb} \\
\end{array}$ & $\begin{array}{c}J / \psi \\
0.1<x_{F}<0.8\end{array}$ & $0.85 \pm 0.008$ & 20 \\
\hline$p, 800$ & $\begin{array}{c}\mathrm{d}, \mathrm{C}, \mathrm{Ca} \\
\mathrm{Fe}, \mathrm{W}\end{array}$ & $\begin{array}{c}J / \psi \text { or } \psi^{\prime} \\
0.15<x_{F}<0.65\end{array}$ & $0.92 \pm 0.008$ & 21 \\
\hline$p, 800$ & $\begin{array}{c}\mathrm{d}, \mathrm{C}, \mathrm{Ca} \\
\mathrm{Fe}, \mathrm{W}\end{array}$ & $\begin{array}{c}\Upsilon \\
0<x_{F}<0.6 \\
p_{T}<4 \mathrm{GeV} / \mathrm{c}\end{array}$ & $0.962 \pm 0.014$ & 22 \\
\hline$p, 800$ & $\begin{array}{c}\mathrm{d}, \mathrm{C}, \mathrm{Ca}, \\
\mathrm{Fe}, \mathrm{W}\end{array}$ & $\begin{array}{c}\Upsilon^{\prime} \text { or } \Upsilon^{\prime \prime} \\
0<x_{F}<0.6 \\
p_{T}<4 \mathrm{GeV} / \mathrm{c}\end{array}$ & $0.948 \pm 0.020$ & 22 \\
\hline
\end{tabular}


Table 3 - The production rate, $N(b \bar{b}) / y$ and $N_{i n t} / s$, for various extcrnal targets having a length of $0.2 \mathrm{~cm}$, assuming the existence of an external beam of $10^{8} \mathrm{p} / \mathrm{s}$. Here $\left\langle l_{i}\right\rangle$ and $\left\langle l_{r}\right\rangle$ are the average interaction and radiation length, respectively, of various targets. The luminosity is denoted by $L$ whereas $R(A)=\sigma^{A}(b \bar{b}) / \sigma_{T}^{A}$ are calculated with $\alpha_{T}=0.72$ and $\alpha(B)=1$. The $P(m)$ quantity represents the probability of having $m$ interactions per bunch crossing.

\begin{tabular}{|c|c|c|c|c|}
\hline & $\begin{array}{c}\mathrm{Be} \\
\mathrm{A}=9\end{array}$ & $\begin{array}{c}\mathrm{Si} \\
\mathrm{A}=28\end{array}$ & $\begin{array}{c}\mathrm{Cu} \\
\mathrm{A}=63\end{array}$ & $\begin{array}{c}\mathrm{W} \\
\mathrm{A}=184\end{array}$ \\
\hline$<l_{i}>, \mathrm{cm}$ & 40.6 & 45.5 & 15.0 & 9.6 \\
$<l_{r}>, \mathrm{cm}$ & 35.2 & 9.4 & 1.4 & 0.35 \\
$L, c n^{-2} s^{-1}$ & $310^{30}$ & $210^{30}$ & $1.710^{30}$ & $1.610^{30}$ \\
\hline$N_{i n t} / s$ & $610^{5}$ & $5.510^{5}$ & $1.710^{6}$ & $2.710^{6}$ \\
$N(b \bar{b}) / y$ & $2.210^{8}$ & $2.810^{8}$ & $1.710^{9}$ & $2.410^{9}$ \\
$P(2) / P(1)$ & $0.610^{-2}$ & $0.510^{-2}$ & $1.610^{-2}$ & $2.510^{-2}$ \\
$P(3) / P(1)$ & $0.210^{-4}$ & $0.1710^{-4}$ & $1.710^{-4}$ & $4.210^{-4}$ \\
$\langle n(p A)\rangle /\langle n(p p)\rangle$ & 1.43 & 1.77 & 2.10 & 2.65 \\
\hline $\mathrm{R}(\mathrm{A})$ & $1 / 27 \mathrm{~K}$ & $1 / 20 \mathrm{~K}$ & $1 / 16 \mathrm{~K}$ & $1 / 12 \mathrm{~K}$ \\
\hline
\end{tabular}

Table 4 - The estimates of the $N(b \bar{b}) / y$ production rate for various gas jet targets having a length of $0.8 \mathrm{~cm}$ (along the beam direction) and $\mathrm{P}(2) / \mathrm{P}(1)=0.05$. This leads to $N_{\text {int }} / s=5.510^{6}$ (and to $\mathrm{P}(3) / \mathrm{P}(1)=1.710^{-3}$ ) for all cases. For each target the number of atoms per $\mathrm{cm}^{3}\left(N_{a t} / \mathrm{cm}^{3}\right)$, the luminosity $L$ as well as R(A) have been calculated. Note that the $\mathrm{Xe}(\mathrm{A}=131)$ and $\mathrm{Cs}(\mathrm{A}=133)$ gas are nearly identical.

\begin{tabular}{|c|c|c|c|}
\hline & $\begin{array}{c}\mathrm{H} \\
\mathrm{A}=1\end{array}$ & $\begin{array}{c}\mathrm{Ar} \\
\mathrm{A}=40\end{array}$ & $\begin{array}{c}\mathrm{Xe} \\
\mathrm{A}=131\end{array}$ \\
\hline$N_{a t} / \mathrm{cm}^{3}$ & $2.510^{13}$ & $1.810^{12}$ & $7.510^{11}$ \\
\hline$L, c m^{-2} s^{-1}$ & $5.410^{32}$ & $7.710^{30}$ & $3.310^{30}$ \\
\hline$N(b \bar{b}) / y$ & $1.110^{9}$ & $3.110^{9}$ & $4.310^{9}$ \\
\hline$\langle n(p A)\rangle /\langle n(p p)\rangle$ & 1 & 1.90 & 2.46 \\
\hline $\mathrm{R}(\mathrm{A})$ & $1 / 50 \mathrm{~K}$ & $1 / 18 \mathrm{~K}$ & $1 / 13 \mathrm{~K}$ \\
\hline
\end{tabular}




\section{References}

1) C.S. Barton et al., Phys. Rev. D27, 2580 (1983):

2) S. Fredrikson et al., Phys. Rep. 144, 187 (1987);

3) A.S. Carrol et al,. Phys. Lett. 80B, 319 (1979);

4) F. Fumuro et al., Nucl. Phys. B152, 376 (1979);

5) See for example: An Expression of Interest in a Super Fixed Target Beauty Facility at the SSC, May 25, 1990;

B. Cox, F. Gilman, T. Gottschalk, Physics of the SSC, Snowmass, 1986, eds. R. Donalson, J. Marx, 33 (1986);

6) S.J. Brodsky, P. Hoyer, Phys. Rev. Lett. 63, 1566 (1989);

7) J.W. Cronin et al., Phys. Rev. D11, 3105 (1975);

8) M.I. Adamovich et al., CERN-EP-/89-123 Report (1989), Contributed paper to the International Symposium on Heavy Quark Physics, Cornell University, June 1989; Heavy quark production,

L. Rossi, CERN/EP 89 137, The IX International Conference on Physics in Collisions, Jerusalem, 19-21 June 1989,

L. Rossi, Hadroproduction of charm, CERN/PPE (1991), International Workshop on Quark Gluon Plasma. Signature, 1-4 October 1990;

9) D.Amtreasyan et al., Phys. Rev. D19, 764 (1979);

10) A. Beretvas et al., Phys. Rev. D34, 53 (1986), P. Skubic et al,. Phys. Rev. D18, 3115 (1978);

11) Kam-Biu Luk, Strangeness Production in High Energy in Proton-Nucleus Collisions, FERMILAB-Conf-89/40, presented at Hadronic Matter in Collisions, Tucson, Arizona (1988);

12) S.P.K. Tavernier, Rep. Prog. Phys. 50, 1439 (1987); R.T. Edwards et al., Phys. Rev. D18, 76 (1978);

13) Heavy Flavor Production in Fixed-Target Experiments, J.A. Appel, FERMILABConf-90-173, Xth International Conference on Physics in Collisions, Durham, North Carolina (1990);

14) H. Cobbaert et al., Phys. Let. B191, 456 (1987);

15) H. Cobbaert et a.l., CERN/88-34 (1988);

16) M.E. Duffy et al., Phys. Rev. Lett. 55, 1816 (1985): 
17) Hadron Production of Charm at Fermilab E769, G.A. Alves et al., presented at the DFP Meeting, Houston, January 1990 and XXV Rencontres de Moriond, March 1990;

18) F. Antinori, Results from WA82 on Charm Physics, presented at Aspen, January 11, 1991

19) J. Badier et al., Z. Phys. C20, 101 (1983);

20) S. Kartik et al.,Phys. Rev D41, 1 (1990);

21) The A-Dependence of $J / \psi$ and $\psi^{\prime}$ production at $800 \mathrm{GeV} / \mathrm{c}, \mathrm{D} . \mathrm{M}$. Alde et al., FERMILAB-Pub-90/156, E-772, (1990);

D.M. Alde et al., Phys. Rev. Lett. 64, 2479 (1991);

22) Nuclear Dependence of the Production of $\Upsilon$ resonances at $800 \mathrm{GeV}$, D.M. Alde et al., LA-UR-91-402 (1991), submitted to Phys. Rev. Lett.;

23) The leading $D$ are those which have one quark coming from the beam or the target;

24) M. Agilar-Benitez et al., Z. Phys. C31, 491 (1986);

25) M.M. Block and R.N. Kahn, Nucl. Phys. 188B, 143 (1987);

26) K.J. Foley et al., Proceedings of the Workshop on Experiments, Detectors, and Experimental Areas for the Supercollider, July 7-17, 1987, Berkeley;

27) A. Fridman and A. Snyder, SLAC-PUB-5319, to be published in LHC Workshop Proceedings, Aachen, Germany, October 4-9, 1990;

28) L. Camilleri, Discussion about a gas jet possibility for the LHC project (January 16,1991$)$. A $0.8 \times 0.2 \mathrm{~cm}^{2}$ vertical target can be considered where $0.8 \mathrm{~cm}$ was along the beam direction;

29) M. Macri, Private discussion;

30) M.J. Tannenbaum, International Journal of Mod. Phys. A, Vol 4, No 14, 3377 (1989). 

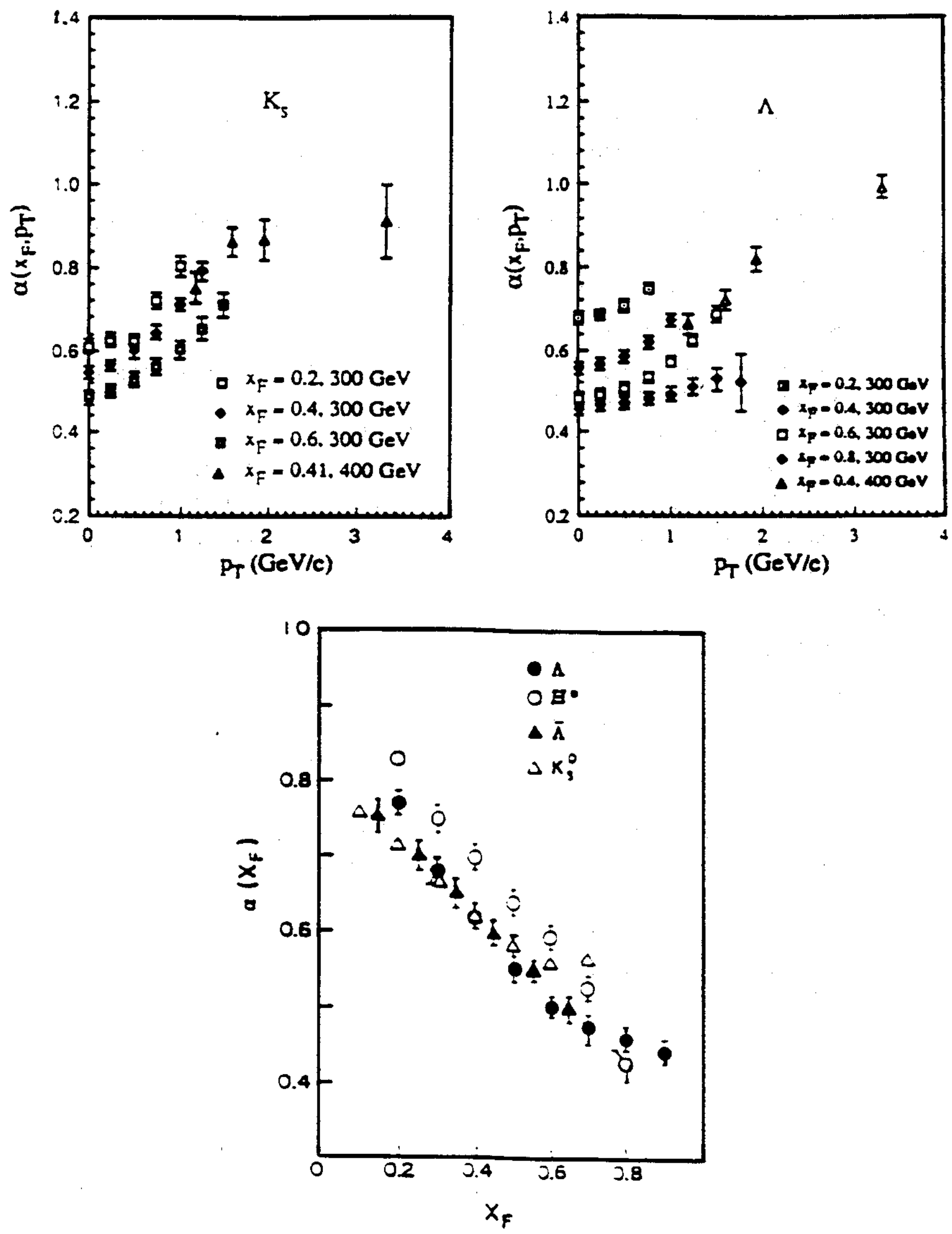

Fig. 1 - The $\alpha\left(x_{F}, p_{T}\right)$ dependance on $x_{F}$ and $p_{T}$ for strange particle production in $p A$ collisions ${ }^{10,11}$. 


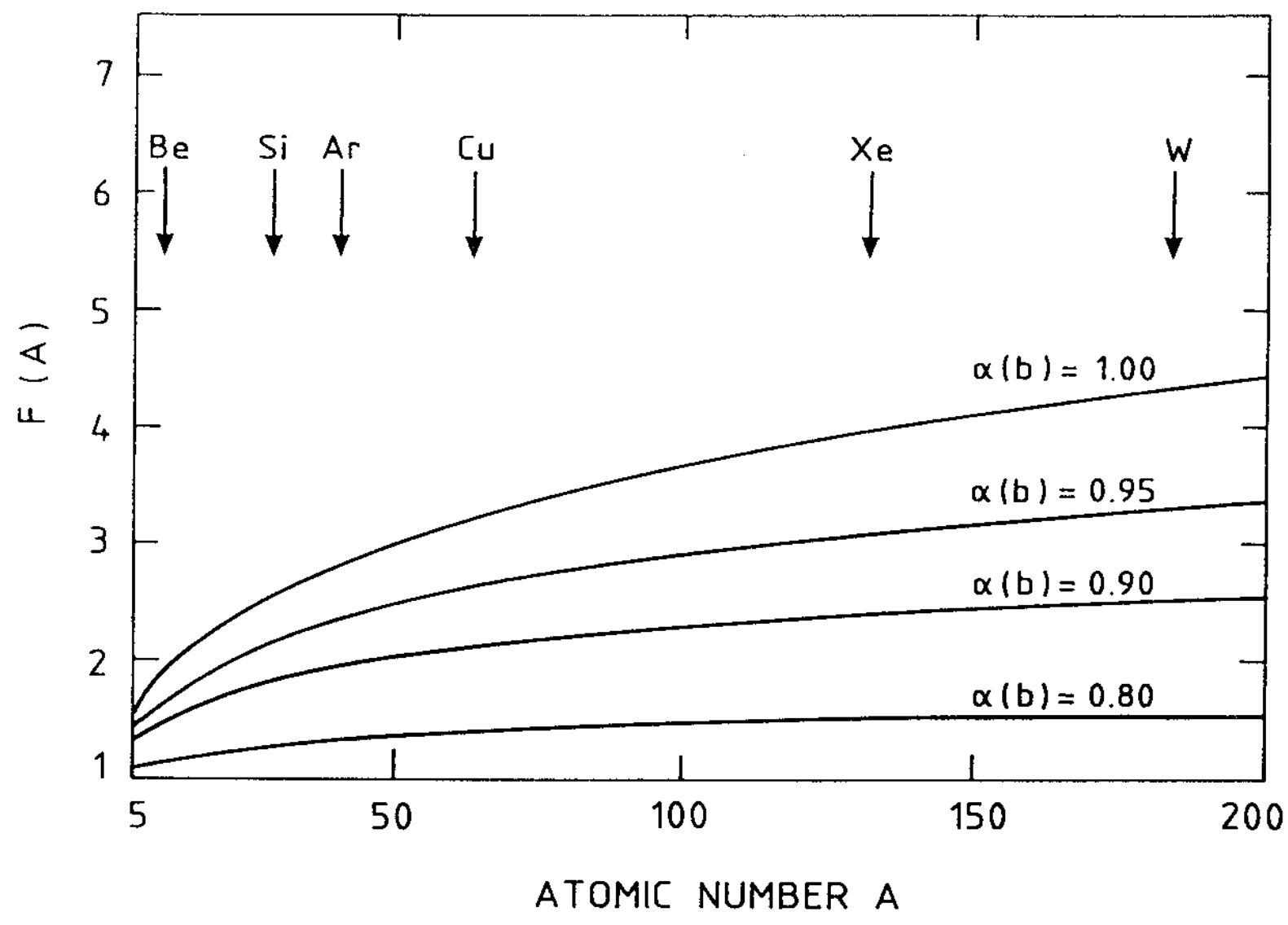

Fig. 2 - Scheme of the $F(A)$ dependence. 